
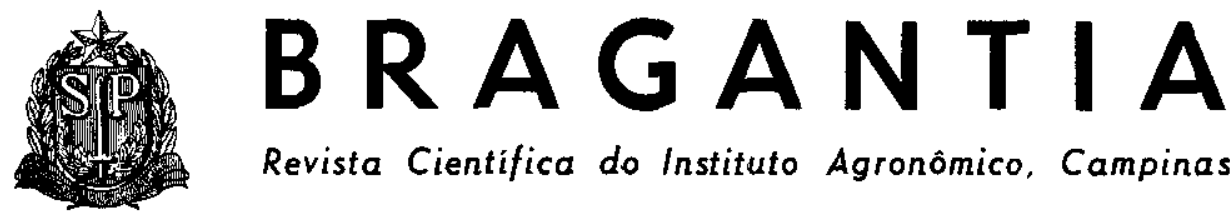

Revista Científica do Instituto Agronômico, Campinas

\title{
EFEITO DA OMISSÃO DE MACRONUTRIENTES EM SISAL (1)
}

Antonio Luiz de Barros Salgado (2), Anísio Azzini, Seção de Plantas Fibrosas; Cen Teixeira Feitosa $\left({ }^{2}\right)$, Seçāo de Fertilidade do Solo, e Rútrer Hiroce, Seção de Química Analítica, Instituto Agronômico

\section{RESUMO}

Plantas de sisal (Agave sisalana Perr.) foram cultivadas em casa de vegetaçáo, em vasos contendo areia lavada e irrigados com solução nutritiva completa e com soluções nutritivas com a omissão de cada macronutriente. As plantas mostraram, na ausência de cada nutriente, as sintomas típicos de sua carência, relacionados com baixos teores do respectivo elemento nas folhas, e redução no seu crescimento e desenvolvimento.

\section{INTRODUÇÃO}

Trabalhos de campo realizados por diversos pesquisadores, entre eles, DOOP $(4,5)$, MEDINA (10), LOC.K (8), COURY (3), BLACK (2) e MAREL (9), demonstraram que dentre os distúrbios fisiológicos que ocorrem na planta de sísal por carência de um nutriente, os mais importantes são os causados pela deficiência de potássio, indicada pelo aparecimento de pequenas manchas pretas na base das folhas; mais tarde, por coalescência, as manchas invadem toda a base da folha, formando um anel preto de tecido necrosado que determina, por estrangu- lamento, a interrupção da circulação da seiva na folha. A folha começa, então, a perder a turgescência, amolece e se enrola no sentido longitudinal até que, no final, se dobra para trás pela parte necrosada, ficando a sua extremidade apoiada no solo.

A carência de outros elementos apresenta os seguintes quadros sintomáticos, descritos pelos autores citados: nitrogênio: clorose nas folhas e plantas cloróticas, com secamento súbito e típico da extremidade das folhas; fósforo: enrolamento, avermelhamento e secamento da extremidade das folhas; condições ácidas do solo:

(1) Apresentado no XVIII Congresso Brasileiro de Ciencia do Solo, realizado em Salvador (BA), em 1981. Recebido para publicaçăo a 27 de abril de 1981.

(2) Com bolsa de suplementaçăo do CNDCT-CNPq. 
Vol. 41, Art. n. ${ }^{\circ} 13$

matizado clorótico das folhas; baixo teor de cálcio no solo: a podridão do cepo.

O presente trabalho visou verificar o efeito da omissão dos macronutrientes no crescimento, desenvolvimento e composição de nutrientes das folhas da planta. Visou, também, determinar a sintomatologia específica e característica para a deficiência de cada um dos macronutrientes relacionada com os teores dos elementos nas folhas.

\section{MATERIAL E METODOS}

No experimento, instalado a 15/6/78 em Campinas e conduzido em casa de vegetação durante 21 meses, utilizou-se $o$ delineamento experimental de blocos ao acaso com sete tratamentos e cinco repetições.

Os tratamentos estudados foram: completo (solução nutritiva com todos os elementos minerais presentes) e solução nutritiva com omissão, respectivamente, de nitrogênio, fósforo, potássio, cálcio, magnésio e enxofre. O tratamento completo foi repetido dez vezes e os demais, apenas cinco.

A solução nutritiva empregada foi a de HOAGLAND \& ARNON (7), modificada por SARRUGE \& HAAG (11).

O plantio foi feito com Agave sisalana Perr., utilizando-se duas mudas por vaso, com seis folhas e $20 \mathrm{~cm}$ de altura cada muda.

As plantas foram cultivadas em vasos com as dimensões de $30 \mathrm{~cm}$ de diâmetro de boca, com capacidade para $10 \mathrm{~kg}$, contendo areia lavada (sílica), e irrigados com soluções nutri- tivas até março de 1980, quando o ensaio foi encerrado. Nessa ocasião, as plantas foram fotografadas e anotados os sintomas de carência nutricional.

As plantas arrancadas dos vasos foram medidas, dissecadas e pesadas, para obtenção de sua altura, número e peso de folhas, peso do bulbo e de raízes e peso total das plantas.

As amostras foliares foram preparadas e submetidas às determinações de macro e micronutrientes, segundo os métodos descritos por BATAGLIA et alii (1).

\section{RESULTADOS E DISCUSSÃO}

Nos quadros 1 e 2 encontram-se os dados referentes ao desenvolvimento das plantas.

\section{a) Altura da planta}

Os resultados de medição revelaram que a altura média das plantas dos tratamentos com a omissão do potássio e do enxofre não diferiu da do tratamento completo, enquanto a das plantas dos tratamentos com a omissão do $\mathrm{N}$, do $\mathrm{P}$, do $\mathrm{Ca}$, e do $\mathrm{Mg}$, foi significativamente mais baixa, inferior em $51,48,43$ e $20 \%$ respectivamente à do tratamento completo.

\section{b) Número e peso de folhas}

O número de folhas do tratamento completo foi significativamente mais elevado do que o dos demais tratamentos, exceto daquele sem $\mathrm{S}$. As reduções do número de folhas foram $52,42,35$ e $30 \%$ para os tratamentos com a omissão do $\mathrm{N}$, do $\mathrm{Ca}$, do $\mathrm{Mg}$ e do $\mathrm{P}$ respectivamente. 
QUADRO 1 - Número, altura e peso das folhas das plantas de sisal cultivado em soluçāo nutritiva completa e na omissão de cada macronutriente

\begin{tabular}{lccc}
\hline Tratamento & Número & Altura & Peso \\
\hline & & $\mathrm{cm}$ & $\mathrm{g}$ \\
Completo & $40 \mathrm{a}$ & $82 \mathrm{a}$ & $2.668 \mathrm{a}$ \\
Sem N & $19 \mathrm{~d}$ & $40 \mathrm{c}$ & $356 \mathrm{c}$ \\
Sem P & $28 \mathrm{c}$ & $43 \mathrm{c}$ & $709 \mathrm{c}$ \\
Sem K & $35 \mathrm{~b}$ & $81 \mathrm{a}$ & $1.693 \mathrm{~b}$ \\
Sem Ca & $23 \mathrm{~d}$ & $47 \mathrm{c}$ & $346 \mathrm{~d}$ \\
Sem Mg & $26 \mathrm{~cd}$ & $65 \mathrm{~b}$ & $716 \mathrm{c}$ \\
Sem S & $37 \mathrm{ab}$ & $76 \mathrm{a}$ & $1.987 \mathrm{~b}$ \\
D.M.S. Tukey 5\% & 4 & 10 & 366 \\
C.V. \% & 2,0 & 11,8 & 19,6 \\
\hline
\end{tabular}

QUADRO 2 - Peso de bulbo, raizes e total das plantas de sisal cultivado ema solução nutritiva completa e na omissão de cada macronutriente

\begin{tabular}{|c|c|c|c|}
\hline \multirow{2}{*}{ Tratamento } & \multicolumn{3}{|c|}{ Peso Médio } \\
\hline & Bulbo & Raízes & Total \\
\hline Completo & $733 a$ & $232 a b$ & $3.633 a$ \\
\hline Sem N & $153 \mathrm{c}$ & $272 a b$ & $781 \mathrm{c}$ \\
\hline Sem P & $207 \mathrm{c}$ & $183 \mathrm{~b}$ & $1.099 \mathrm{c}$ \\
\hline Sem $\mathbf{K}$ & $340 \mathrm{~b}$ & $314 a$ & $2.347 \mathrm{~b}$ \\
\hline Sem Ca & $94 \mathrm{~d}$ & $171 \mathrm{~b}$ & $611 \mathrm{c}$ \\
\hline Sem $\mathbf{M g}$ & $138 \mathrm{c}$ & $155 \mathrm{~b}$ & $1.009 \mathrm{c}$ \\
\hline Sem $\mathbf{S}$ & $420 \mathrm{~b}$ & $336 a$ & $2.743 b$ \\
\hline D.M.S. Tukey 5\% & 109 & 122 & 539 \\
\hline c.V. \% & 22,7 & 36,2 & 19,4 \\
\hline
\end{tabular}


Resultados similares foram obtidos em relação ao peso das folhas. As omissões do nitrogênio e do cálcio foram os tratamentos que apresentaram maiores reduções no peso das folhas. Essas reduções foram respectivamente $47,30,12,42,35 \mathrm{e}$ $8 \%$ para os tratamentos com omissão de $\mathrm{N}, \mathrm{P}, \mathrm{K}, \mathrm{Ca}, \mathrm{Mg}$ e $\mathrm{S}$.

\section{c) Peso dos bulbos}

Verificou-se que o tratamento completo apresentou peso de bulbo significativamente superior ao dos demais tratamentos. A redução de peso em relação ao tratamento completo foi $87,81,79,72,54$ e $43 \%$ respectivamente para os tratamentos com a omissão de $\mathrm{Ca}, \mathrm{Mg}, \mathrm{N}, \mathrm{P}$, $\mathrm{K}$ e $\mathrm{S}$.

\section{d) Peso das raízes}

Com relação ao peso das raízes, - tratamento completo não diferiu significativamente dos demais. $\mathrm{O}$ coeficiente de variação $(36 \%)$ foi mais elevado que o dos demais parâmetros avaliados.

\section{e) Peso total da planta}

Os resultados demonstraram que o peso total das plantas do tratamento completo foi significativamente mais elevado do que o dos demais tratamentos. A redução de peso foi $83,79,73$ e $70 \%$ respectivamente para os tratamentos com a omissão de $\mathrm{Ca}, \mathrm{N}, \mathrm{Mg}$ e $\mathrm{P}$.

\section{f) Análise química das folhas}

Pelos quadros 3 e 4, nota-se que as quedas dos teores de macronutrien-

QUADRO 3 - Teores de N, P e K nas folhas de sisal cultivado em solução nutritiva e com a omissão de cada macronutriente

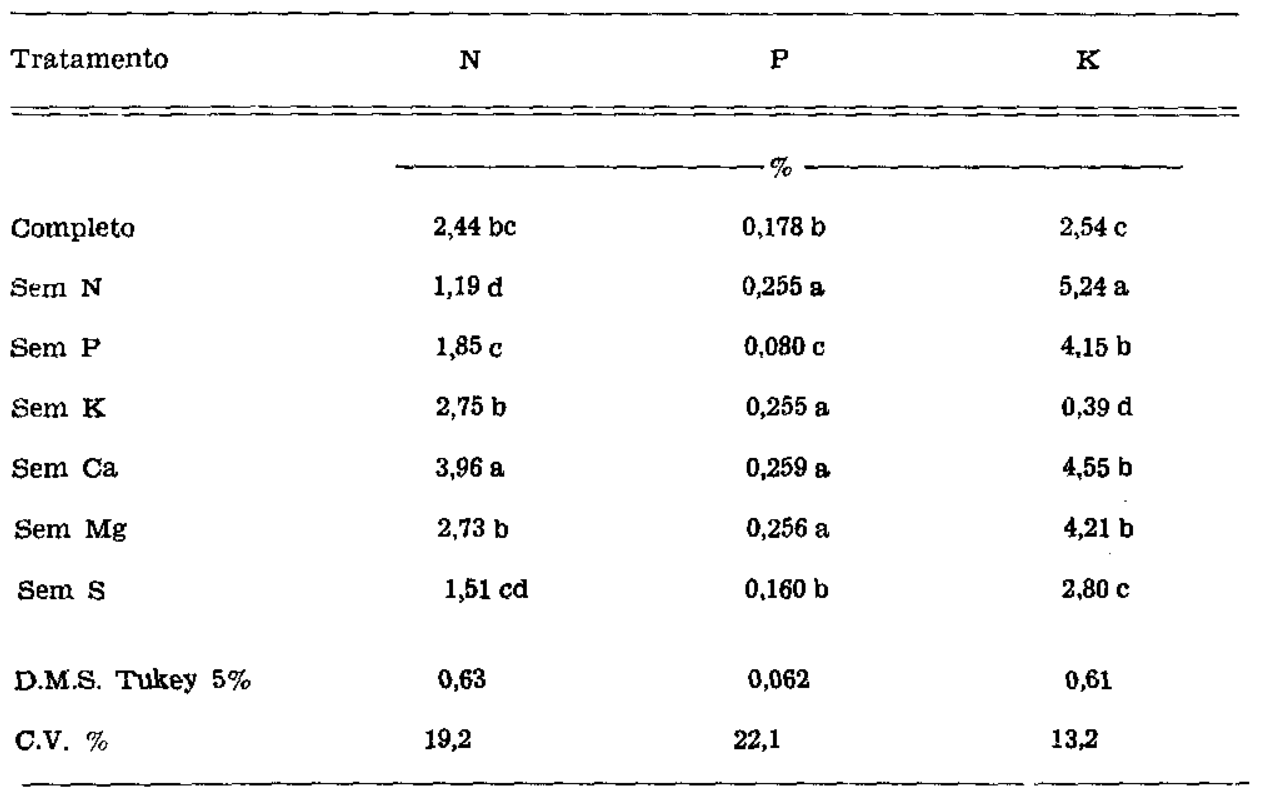


tes das folhas, quando se compara o tratamento completo com os tratamentos com a omissão dos diversos nutrientes, foram mais intensas para o potássio $(2,54$ para $0,39 \%)$, cálcio $(0,80$ para $0,16 \%)$ e enxofre $(0,08$ para $0,02 \%$ ); as quedas nos teores de nitrogênio $(2,44$ para $1,19 \%)$, fósforo $(0,178$ para $0.080 \%)$ e magnésio $(0,38$ para $0,167 \%)$ foram menores, em torno de duas e meia vezes.

Pode-se notar que o tratamento completo não foi o que apresentou teores mais elevados de todos os macronutrientes nas folhas. Assim, o tratamento com a omissão de Ca apresentou teor de $\mathrm{N}$ mais elevado; os teores de $\mathrm{P}$ mais elevados foram apresentados pelos tratamentos com omissão de $\mathrm{N}, \mathrm{K}, \mathrm{Ca}$ e $\mathrm{Mg}$; o teor mais alto de $\mathrm{K}$ e de $\mathrm{C}$ foi notado com omissão de $\mathrm{N}$; o de $\mathrm{Mg}$, com omissão de $\mathrm{K}$ e, o de $\mathrm{S}$, de $\mathrm{Ca}$.

Em relação aos micronutrientes, apesar de serem aplicados em doses iguais em todos os tratamentos, os teores nas folhas variaram de duas a quatro vezes. $O$ teor de boro variou de $78 \mathrm{ppm}$ (com omissão de N) para 29 ppm (omissão de S); o de cobre, de 3,6 ppm (omissão de N) a $1,0 \mathrm{ppm}$ (omissão de P); o de ferro, de $168 \mathrm{ppm}$ (omissäo de $\mathrm{Ca}$ ) a $86 \mathrm{ppm}$ (omissão de P); o de manganês, de $67 \mathrm{ppm}$ (omissão de Ca) a $33 \mathrm{ppm}$ (omissão de P); o de zinco, de 36,2ppm (omissão de N) a 14,7 ppm (omissão de $\mathrm{Mg}$ ).

\section{g) Descrição dos sintomas de deficiências}

Nitrogênio: Plantas atrofiadas, pequeno porte, clorose generalizada nas folhas, conforme o constatado por BLACK (2) em solos com baixo teor de nitrogênio. Há ainda necrose e séca nas extremidades das folhas próximas da base. Plantas com secamento súbito e típico da extremidade das folhas são encontradas em solos deficientes de nitrogênio $(5,6)$.

QUADRO 4 - Teores de $\mathrm{Ca}, \mathrm{Mg}$ e $\mathrm{S}$ nas folhas de sisal cultivado em solução nutritiva completa e com a omissāo de cada nutriente

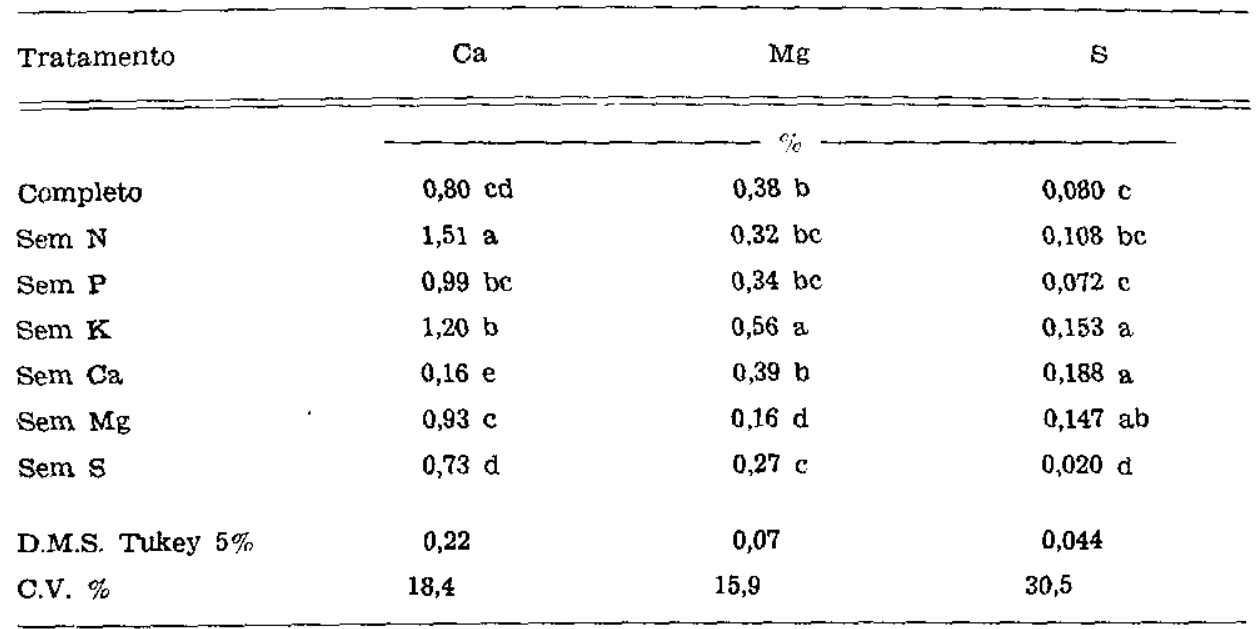


$\mathrm{Na}$ figura 1, observam-se plantas tratadas com a omissão de nitrogênio em comparação com as do tratamento completo.

Fósforo: Pequeno desenvolvimento das plantas e intensa coloração esverdeada das folhas.

$\mathrm{Na}$ figura 2, observam-se plantas tratadas com a omissão de fósforo em comparação com as do tratamento completo.

Potássio: Nécrose na base das folhas e séca nas extremidades destas, similares ao descrito por DOOP (6), MEDINA (10), LOCK (8) e COURY (3).

$\mathrm{Na}$ figura 3, observam-se plantas tratadas com omissão de potássio comparadas com as do tratamento completo.

Cálcio: Plantas praticamente arrasadas pelo matizado clorótico das folhas e pela necrose, similares aos observados por BLACK (2). As fo- lhas necrosadas secam da base para os ponteiros, dobrando-se para o chão, até secarem totalmente.

$\mathrm{Na}$ figura 4, observam-se plantas tratadas com omissão de cálcio comparadas com as do tratamento completo.

Magnésio: Plantas fracas, folhas finas, clorose generalizada, folhas $\mathrm{da}$ base moles dobradas para o chão. MAREL (9) relata a ocorrência de sintomas da deficiência de magnésio em plantações situadas em solos arenosos de turfas liparíticos de Sumatra.

$\mathrm{Na}$ figura 5 , observam-se plantas tratadas com a omissão de magnésio e comparadas às do tratamento completo.

Enxofre: Plantas amareladas, clorose generalizada fraca e necroses ocasionais na base das folhas.

Na figura 6, observam-se plantas tratadas com omissão de enxofre comparadas com as do tratamento completo.

QUADRO 5 - Teores de micronutrientes nas folhas de sisal cultivado em solução nutritiva completa e com a omissāo de cada nutriente

\begin{tabular}{lccccc}
\hline Tratamento & B & Cu & Fe & Mn & Zn \\
\hline & $37 \mathrm{a}$ & $2,1 \mathrm{a}$ & $111 \mathrm{a}$ & $36 \mathrm{a}$ & $16 \mathrm{a}$ \\
Completo & $78 \mathrm{~b}$ & $3,6 \mathrm{~b}$ & $123 \mathrm{ab}$ & $60 \mathrm{~b}$ & $36 \mathrm{~b}$ \\
Sem N & $59 \mathrm{ab}$ & $1,0 \mathrm{a}$ & $86 \mathrm{a}$ & $33 \mathrm{a}$ & $19 \mathrm{a}$ \\
Sem P & $66 \mathrm{ab}$ & $1,9 \mathrm{a}$ & $107 \mathrm{a}$ & $38 \mathrm{a}$ & $16 \mathrm{a}$ \\
Sem K & $42 \mathrm{a}$ & $1,3 \mathrm{a}$ & $168 \mathrm{~b}$ & $67 \mathrm{~b}$ & $27 \mathrm{ab}$ \\
Sem Ca & $56 \mathrm{ab}$ & $2,5 \mathrm{ab}$ & $117 \mathrm{ab}$ & $39 \mathrm{a}$ & $15 \mathrm{a}$ \\
Sem Mg & $29 \mathrm{a}$ & $3,1 \mathrm{ab}$ & $102 \mathrm{a}$ & $38 \mathrm{a}$ & $16 \mathrm{a}$ \\
Sem S & 29 & 1,1 & 55 & 19 & 9,2 \\
D.M.S. Tukey $5 \%$ & 40,9 & 36,5 & 34,6 & 32,8 & 32,6 \\
C.V. \% & & & & &
\end{tabular}




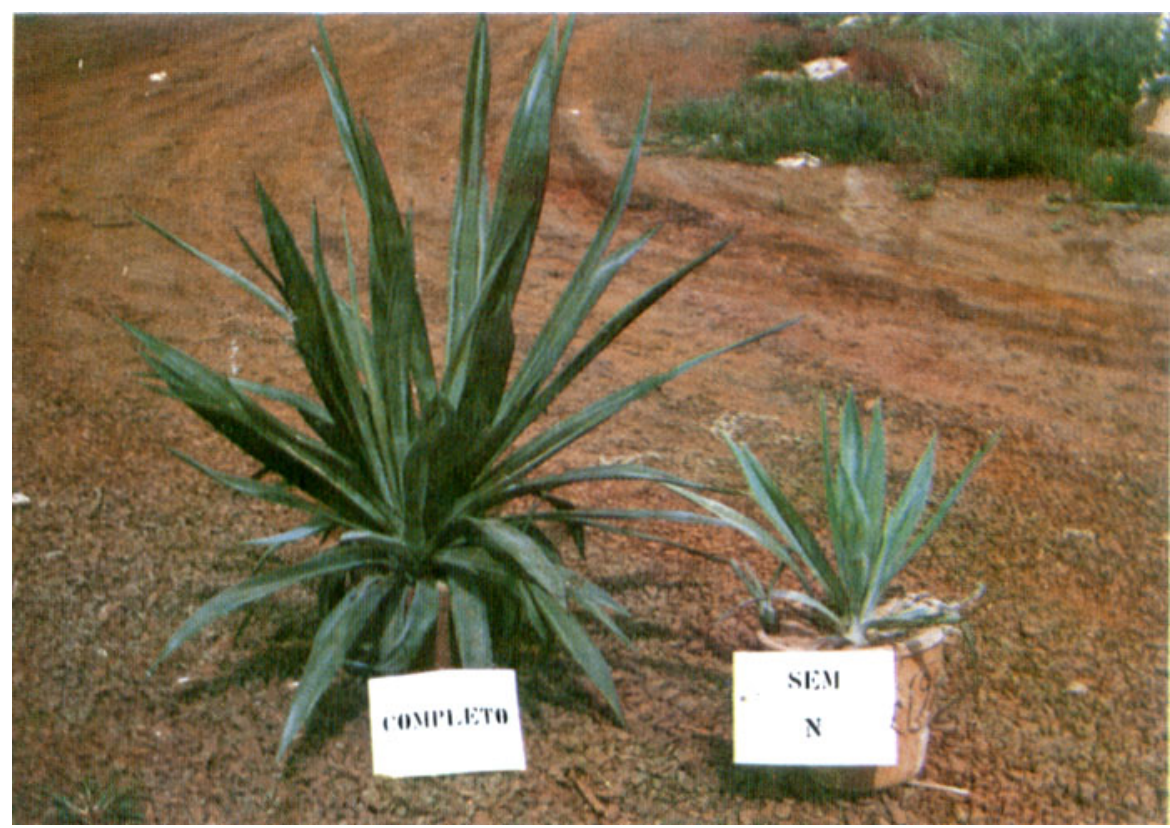

Figura 1. -- Plantas tratadas com omissăo de nitrogènio em comparaçāo com as do tratamento completo

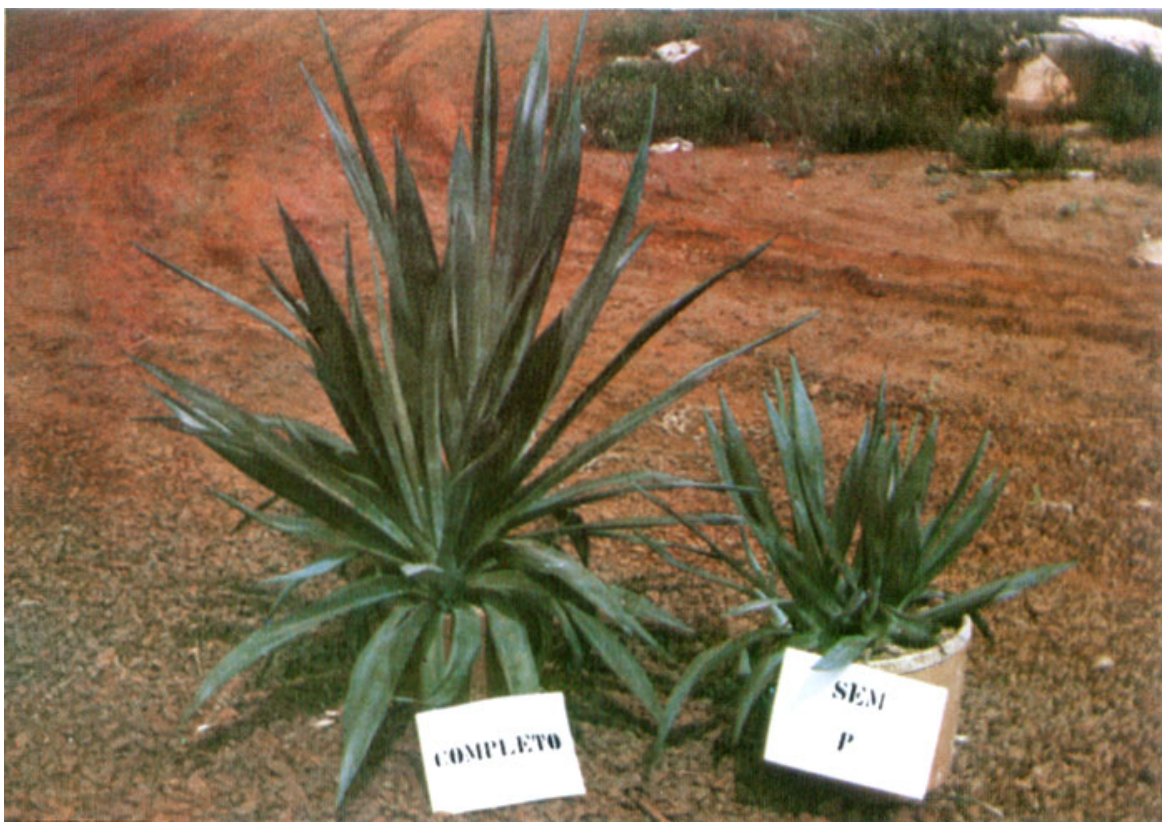

Figura 2. - Plantas tratadas com omissão de fósforo em comparạ̧ăo com as do tratamento completo 


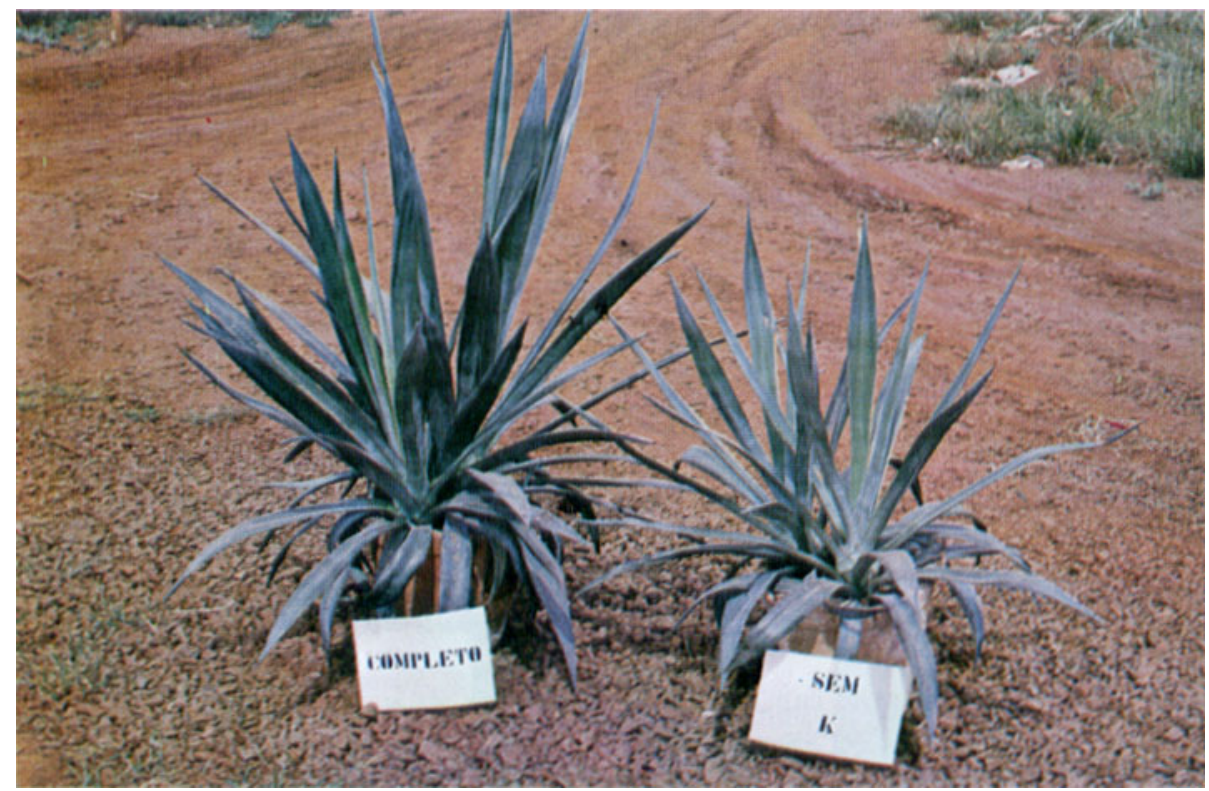

Figura 3. - Plantas tratadas com omissão de potássio comparadas com as do tratamento completo

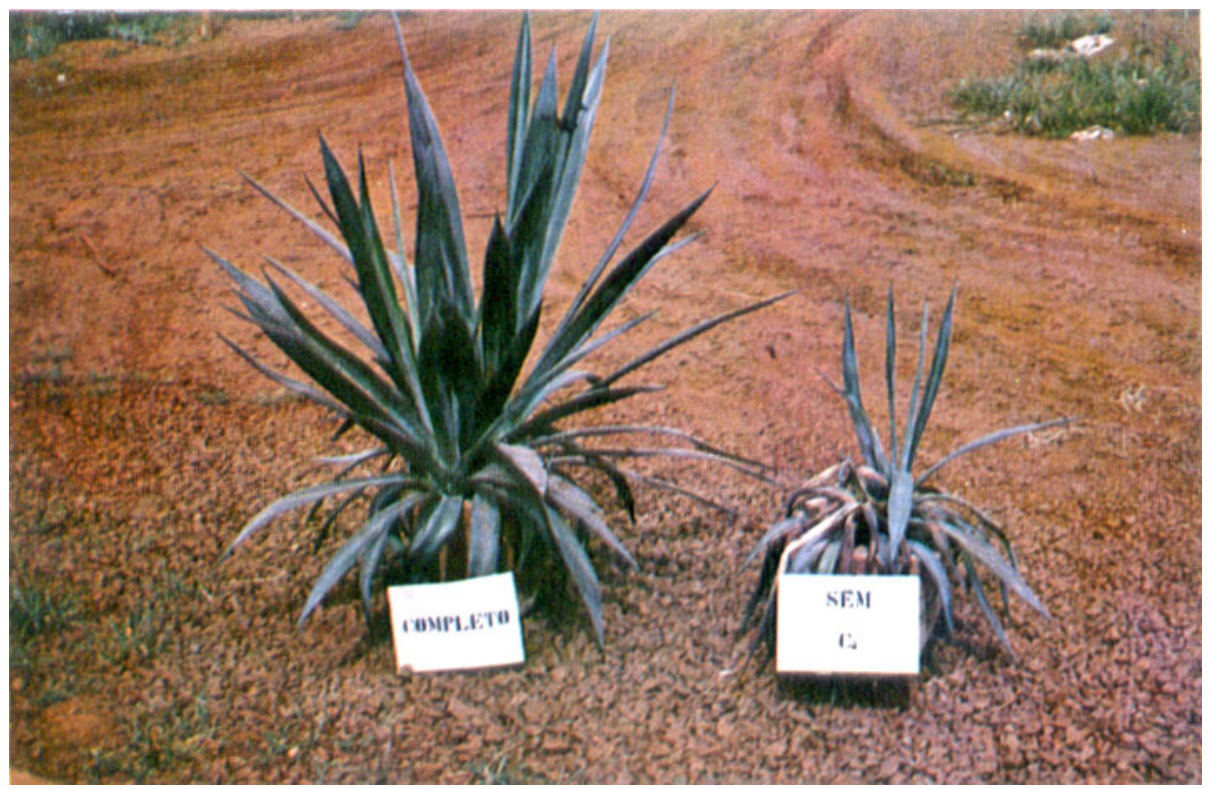

Figura 4. - Plantas tratadas com omissão de cálcio comparadas com as do tratamento compieto 


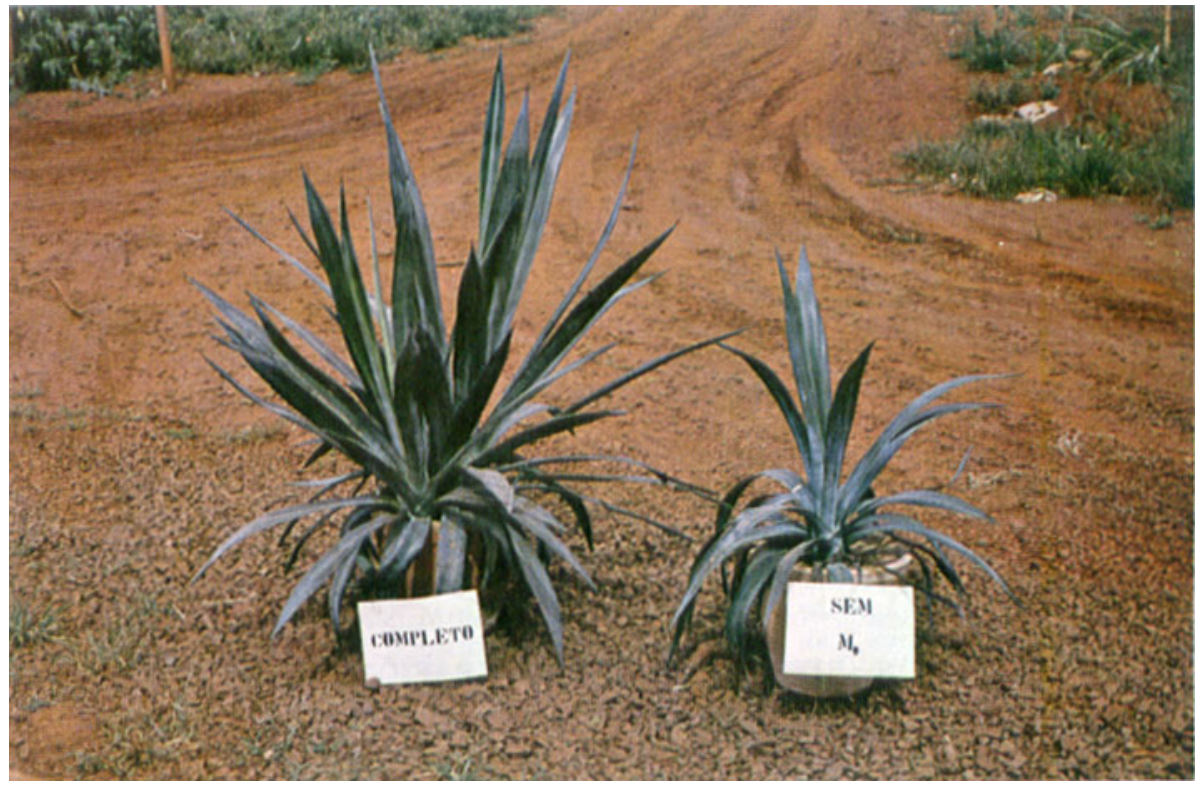

Figura S. - Plantas tratadas com omissro de magnesio comparadas com as do tratamento completo

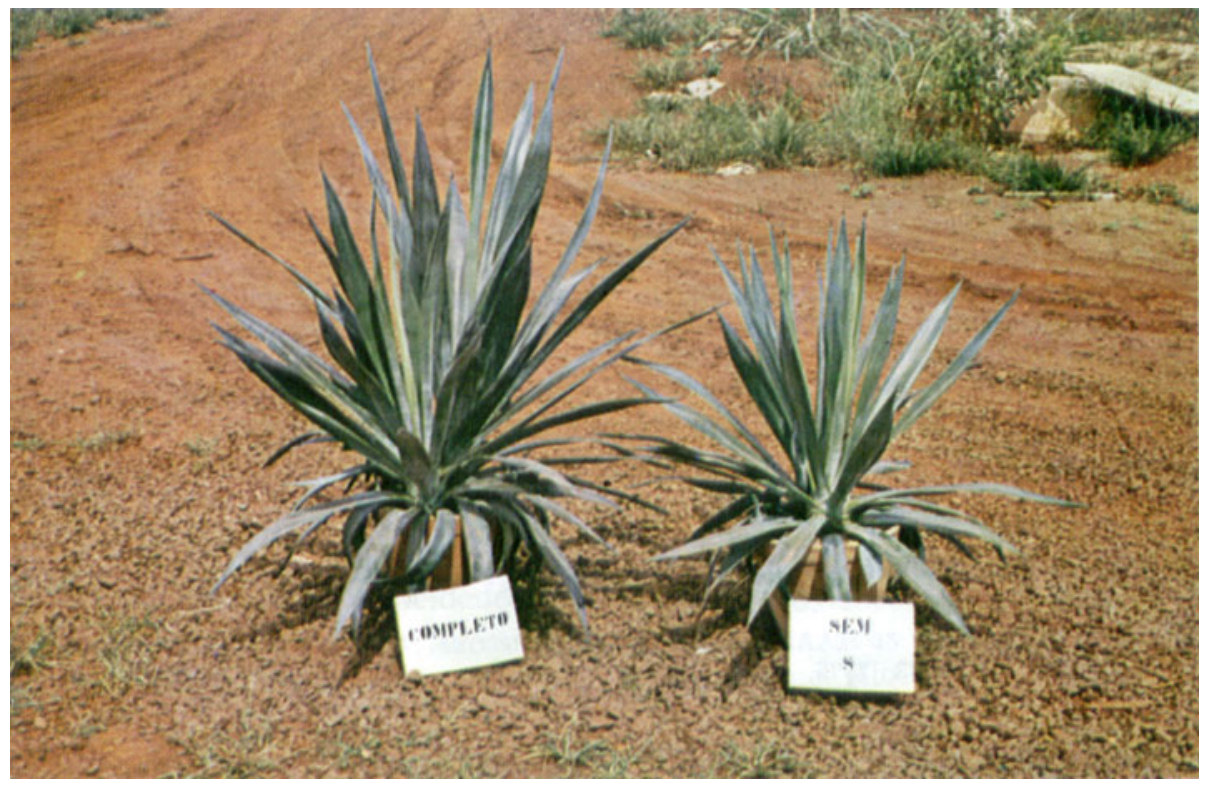

Figuta 6 . - Plantas tratadas com omissâo de enxofire comparadas com as do tratamento completo 


\section{CONCLUSÕES}

A omissão de macronutrientes na solução nutritiva acarretou redução no crescimento da planta, no peso das folhas, bulbo e raizes, provocou aparecimento de sintomas típicos da deficiência do respectivo nutriente relacionada a teores mais baixos do respectivo elemento nas folhas do sisal $\mathrm{e}$ influenciou absorção de micronutrientes pela planta.

\section{MACRONUTRIENTS DEFICIENCY ON SISAL (AGAVE SISALANA PERR,)}

\section{SUMMARY}

Sisal plants were cultivated in washed sand during twenty-one months. The plants were irrigated with complete nutrient solution and solutions with absence of each macronutrient ( $\mathrm{N}, \mathrm{P}, \mathrm{K}, \mathrm{Ca}, \mathrm{Mg}$ e $\mathrm{S}$ ). The plants, in the absence of each macronutrient, showed typical symptoms which were related to the low level of the respective element in the leaf. The growing of the leaves was reduced by the absence of the macronutrients.

\section{REFERENCIAS BIBLIOGRAFICAS}

1. BATAGLiA, O. C.; TELXEIRA, J.P.F.; FURLANI, P. R.; FURLANI, A. M. C.; GALLO, J. R. Métodos de análise química de plantas. Campinas, Instituto Agronômico, 1978. 31p. (Circular, 87)

2. BLACK, I. A. Report of a preliminary survey of sisal diseases in Tanganyika Territory: N. 4. Tanganyika Territory Sisal Research Station, 1948. 30p.

3. COURY, T. Em torno da questão "deficiências minerais em sisal e a necrose da base das folhas". Anais da Escola Superior de Agricultura "Luiz de Queiroz", Piracicaba, 8:387-398, 1951.

4. DOOP, J. E. A. den. The utilization of sisal waste in Java and Sumatra. Part. III. East African Agricultural Journal, 4:343-351, 1939.

5. The utilization of sisal waste in Java and Sumatra. Part. IV. East African Agricultural Journal, 4:415-425, 1939.

6. The utilization of sisal waste in Java and Sumatra. Part. V. East African Agricultural Journal, 5:312-320, 1940.

7. HOAGLAND, D. R. \& ARNON, D. I. The water culture method for growing plants without soil. Berkeley, California Agricultural Experiment Station, 1950, 32p. (Circular, 347)

8. LOCK, G. W. Sisal-Tropical: Thirty year's sisal research in Tanganica. 2. ed. London, Longmans, 1969. 365p. (Agriculture series)

9. MAREL, A. W. van der. Het beschikbaar komen van Kalium en Magnesium voor de plant in boden mineralen by verschillende grondsoorten. Landbouw-Kundig Tydschrift, 3:178-189, 1950 .

10. MEDINA, J. C. A necrose da base da folha de sisal. Bragantia, Campinas, 3:73-84, 1943.

11. SARRUGE, J. R. \& HAAG, P. Soluçōes nutritivas. Summa Phytopatologica, Piracicaba, 1:231-233, 1975. 